\title{
Pathophysiology and underlying mechanisms in hereditary angioedema
}

\author{
Alberto López Lera
}

Centre for Biomedical Network Research on Rare Diseases (CIBERER) U-754, Hospital La Paz Institute for Health Research (IdiPaz), Madrid, Spain

This review aims to summarize the main pathophysiological events involved in the development of hereditary angioedema (OMIM\#106100). Hereditary angioedema is a rare genetic disease inherited in an autosomal dominant manner and caused by a loss of control over the plasma contact system or kallikrein-kinin system, which results in unrestrained bradykinin generation or signaling. In patients with hereditary angioedema, BK binding to endothelial cells leads to recurrent episodes of swelling at subcutaneous or submucosal tissues that can be life threatening when affecting the upper respiratory tract. The disease can either present with hypocomplementemia owing to the presence of pathogenic variants in the gene encoding complement $\mathrm{C} 1$ inhibitor (hereditary angioedema with C1-inhibitor deficiency) or present with normocomplementemia and associate with elevated estrogen levels owing to gain-of-function variants in the genes encoding coagulation proteins involved in the kallikrein-kinin system (namely, coagulation FXII [FXII-associated hereditary angioedema], plasminogen [PLG-associated hereditary angioedema], and high-molecular-weight kininogen [KNG1-associated hereditary angioedema]). Moreover, in recent years, novel pathogenic variants have been described in the genes encoding angiopoietin 1 ( $A N G P T 1$-associated hereditary angioedema) and myoferlin ( $M Y O F$-associated hereditary angioedema), which further expand the pathophysiological picture of hereditary angioedema.
Patients with hereditary angioedema (HAE) (OMIM\#106100) experience chronically recurrent episodes of swelling localized to the subcutaneous or submucosal tissues that result from periodic increases in vascular permeability. Edema attacks in HAE are nonpruritic and self-limited with ill-defined margins and can affect different anatomic sites (mainly the face, extremities, genitals, respiratory tract, and intestinal or mesenterial structures). These episodes have usually a slow onset and relapse, resolving spontaneously within 48 to 72 hours in most of the cases. HAE is a complex genetic disease inherited in an autosomal dominant manner with a prevalence estimated in 1-9 of 100,000. ${ }^{1}$ The most common and historically first recognized form of HAE is that caused by the functional deficiency of the serpin $\mathrm{C} 1$ inhibitor $(\mathrm{C} 1-$ INH) (HAE with C1-INH deficiency [HAE-C1-INH]), ${ }^{2}$ but nonhypocomplementemic, estrogen-related disease subtypes (HAE with normal C1-INH levels [HAE-nC1-INH]) have more recently been described, which are due to heterozygous variants affecting coagulation factor FXII (HAE-FXII), PLG (HAE-PLG), ANGPT1 (HAE-ANGPT1), and high molecular KNG1 (HAE-KNG1) genes. Moreover, the genetic defect(s) responsible for the disease in a subset of patients with HAE-nC1INH are still unknown (HAE unknown [HAE-U]). ${ }^{3}$

Edema in HAE originates from a loss of control over the plasma contact (CAS) or kallikrein-kinin (KKS) system, which results in unrestrained bradykinin (BK) generation or signaling. Research developed during the last 2 decades has significantly widened our understanding of HAE pathophysiology and provided meaningful insights into the biological activities exerted by the KKS. This review aims to give a concise account of our present knowledge about the pathogenic mechanisms of HAE.

\section{Contact activation of FXII and the plasma kallikrein-kinin}

The KKS is a proteolytic cascade that drives proinflammatory and procoagulant pathways. It is comprised of the protease precursors prekallikrein (PK) (or Fletcher factor), coagulation factors XI (FXI) and XII (FXII) (or Hageman factor), and the cofactor/substrate high-molecular-weight kininogen (HK). ${ }^{4,5} \mathrm{PK}$ and FXI each form circulating complexes with HK (at stoichiometries of 1:1 and $1: 2$, respectively) by binding to overlapping sites on $\mathrm{HK}$.

FXII is the zymogen of the serine protease FXIIa. It is secreted by hepatocytes as a 596 amino acid, $80 \mathrm{kDa}$ single-chain protein at a plasma concentration of $40 \mu \mathrm{g} / \mathrm{ml}(375 \mathrm{nM}) .{ }^{6}$ FXII presents a modular architecture of protein domains consisting of the following (in $\mathrm{N}$-ter to $\mathrm{C}$-ter orientation): a type 2 fibonectin domain, 2 EGF-like domains, 1 type I fibronectin domain, 1 kringle domain, 1 proline-rich domain, and a peptidase-type catalytic domain. ${ }^{7}$ The binding of FXII to target surfaces is mediated by the second EGFlike domain and the kringle domain.

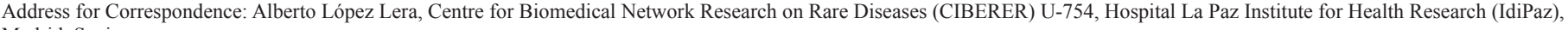
Madrid, Spain

e-mail: alberto.lopez@ciberer.es

Received: October 26, 2020 Accepted: November 12, 2020 •DOI: 10.4274/balkanmedj.galenos.2020.2020.10.166

Available at www.balkanmedicaljournal.org

ORCID iD of the author: A.L-L. 0000-0002-9596-6910.

Cite this article as:

López Lera A. Pathophysiology and underlying mechanisms in hereditary angioedema. Balkan Med J. 2021;38(2):82-88.

Copyright@Author(s) - Available online at http://balkanmedicaljournal.org/ 
In contact with negatively charged surfaces, FXII undergoes autoactivation, a process by which conformational changes induce zymogen-enzyme conversions through surface-catalyzed biochemistry. It is now widely accepted that such conformational changes turn FXII into a substrate for traces of circulating enzymes. ${ }^{8}$ This process constitutes the contact activation phase of the KKS and results in the cleavage of FXII after Arg343 and Arg353 residues, which releases activated FXII (FXIIa) (Figure 1).

FXIIa has a molecular weight of $82 \mathrm{kDa}$ and is composed of a 50 $\mathrm{kDa}$ heavy-chain disulfide linked to a $32 \mathrm{kDa}$ light chain that contains the active site. FXIIa preserves the surface-binding domains of FXII, which mediates its clotting activity. A second cleavage after R334 converts FXIIa into FXIIf, a 2-chain active enzyme with an approximated molecular weight of 28-30 kDa. This eliminates the domains that are essential for binding to prothrombotic surfaces, and therefore, FXIIf does not have the capacity to act as a clotting factor but only as a fluid-phase KKS activator. Instead, FXIIf can activate the complement system by cleaving the complement $\mathrm{C} 1$ in a plasmin-independent manner, which probably contributes to local complement consumption in patients with HAE-C1INH. ${ }^{9}$

Both FXIIa and FXIIf can cleave the 638 amino acid, $84 \mathrm{kDa}$ single-chain zymogen PK to release plasma kallikrein (PKa), a serine protease consisting of a $50 \mathrm{kDa}$ heavy-chain disulfide linked to a light chain of $33 \mathrm{kDa} .{ }^{10}$ Most PK molecules $(85 \%)$ circulate,

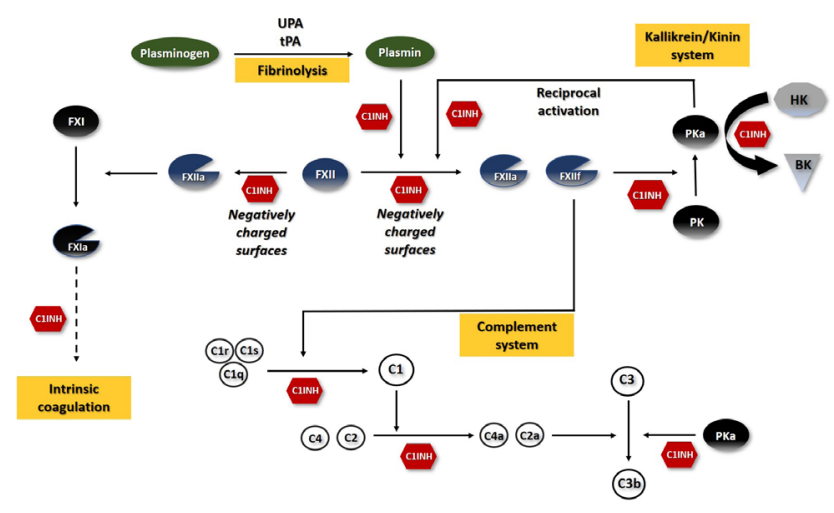

FIG. 1. Enzymatic cascades crosstalk in the pathophysiology of HAE. Uncontrolled activation of the KKS triggers angioedema development in HAE. FXII proenzyme activation by either contact with negatively charged surfaces or enzymatic processing (not shown) releases the active proteases FXIla and FXIIf, which can activate PK to PKa (KKS) and $\mathrm{FXI}$ to $\mathrm{FXla}$ (intrinsic coagulation). PKa in turn activates more FXII molecules and cleaves HK to release BK. FXIIf and PKa also activate the classical pathway of complement respectively at the $\mathrm{C} 1$ and $\mathrm{C} 3 \mathrm{lev}$ els. Plasminogen activation also contributes to HAE pathophysiology. BK can induce fibrinolysis transactivation by promoting plasmin release in UPA or tPA-dependent manners while in turn plasmin activates FXII to FXIla. C1INH keeps tight control of this enzymatic crosstalk by serpin mechanism-mediated inhibition of target proteases (indicated).

$\mathrm{BK}$, Bradykinin; AE, angioedema; C1-INH, C1 esterase inhibitor; FXII, coagulation Factor XII ; FXIla, activated coagulation Factor XII; FXIIf, betha-FXIIa; PK, PlasmaKallikrein; PKa, activated Plasma Kallikrein; HK, High Molecular Weight Kininogen; UPA, Urokinase-tipe Plasminogen Activator; tPA, Tissue Plasminogen Activator. forming 1:1 complexes with the substrate and cofactor HK through which it is recruited to the surface of endothelial cells. ${ }^{11}$ On activation, PKa can reciprocally activate more FXII molecules to FXIIa (by proteolysis of FXII after Arg343 or Arg353). Autoactivation of FXII after contact with activating surfaces is a relatively slow reaction. Reciprocal activation between PK and FXII results in a positive feedback loop that is responsible for the rapid burst of activity seen on KKS activation. ${ }^{12}$ The activation process eventually leads to the cleavage of HK by PKa, which releases BK, the most important kinin in the KKS. PKa can also cleave and activate the complement protein $\mathrm{C} 3$ triggering local inflammation. ${ }^{13}$ (Figure 1).

HK plays a central role in KKS assembly and regulation. Kininogens are multifunction proteins with antithrombotic, antidiuretic, profibrinolytic, antiadhesive, antiangiogenic, and proinflammatory properties. $\mathrm{HK}$ is one of the 2 alternative splicing products of the KNG1 gene (the other one is low-molecular-weight kininogen [LK]) and has a molecular weight of $120 \mathrm{kDa}$. It is secreted by hepatocytes as a plasmatic protein that eventually binds to the endothelial wall mainly through interaction with endothelial cell membrane receptors. After cleavage of HK by PKa after Lys362 and $\operatorname{Arg} 371, \mathrm{BK}$ is released, and $\mathrm{HK}$ is converted into a 2-chain, disulfide-linked protein. ${ }^{14,15}$

The KKS assembles on cell surfaces in a zinc-dependant, saturable, and reversible reaction. Both HK and FXII compete with similar affinities for binding sites on platelets and human umbilical vein endothelial cells. To date, 3 HK-binding proteins have been identified in cellular surfaces: the receptor for the $\mathrm{gC} 1 \mathrm{qR}, \mathrm{CK} 1$, and the u-PAR. ${ }^{16-18}$ These 3 endothelial cell proteins can act separately or combine to form bimolecular complexes with different affinities for HK and FXII that regulate KKS activation and dictate its interrelations with other proteolytic systems. For example, kallikrein cleavage in HK-PK complexes promotes u-PAR-bound pro-urokinase conversion to urokinase, which in turn converts plasminogen to plasmin and initiates fibrinolysis. ${ }^{8}$

Besides the classical, contact-mediated activation driven by FXIIa, the KKS may become activated by noncanonical activators. To date, several of such activators have been identified: plasmin, prolylcarboxypeptidase (PRCP), extracellular HSP90, and misfolded protein aggregates.

In the 1970 s, it was discovered that plasmin can physiologically activate FXII to FXIIa in the fluid phase and that FXIIa in turn cleaves and activates plasminogen to yield active plasmin. ${ }^{19}$ This functional link is probably of high relevance for the pathophysiology of all HAE disease variants, as suggested by recent findings in HAE-FXII and HAE-PLG (see the sections below). PRCP is a highly conserved serine protease with relevant roles as a regulator of cardiovascular functions, such as blood pressure and electrolyte balance. In vitro studies have shown that PRCP is the major PK activator when bound to cells. ${ }^{20,21}$ HSP90 is a ubiquitous protein in eukaryotes and bacteria that functions as an intracellular molecular chaperone assisting in the folding and stabilization of other proteins but that also displays extracellular functions. HSP90 activates $\mathrm{PK}$ to $\mathrm{PKa}$ in a zinc-dependant manner only when both proteins are bound with HK in a trimolecular complex. Because HSP90 
lacks proteolytic activity, its PK-activating properties are probably related to a chaperone effect on the conformation of the PKHK complex that accelerates PK autoactivation. ${ }^{22,23}$ Finally, it has been demonstrated that misfolded protein aggregates formed by denaturation in vitro or those present in the plasma from patients with systemic amyloidosis in vivo activate FXII and PK without activating FXI. ${ }^{24}$

KKS activation is tightly regulated by 3 major enzymes: C1-INH, alpha-2-macroglobulin ( $\alpha 2 \mathrm{M})$, and antithrombin III (ATIII). C1INH is the only major plasma inhibitor of factor XIIa and factor XIIf. ATIII can also inhibit FXIIa, although its contribution is apparently minor under physiological conditions as compared with that of C1-INH. $\alpha 2 \mathrm{M}$ and $\mathrm{C} 1-\mathrm{INH}$ are the main regulators of PK activation and together account for $>90 \%$ of the kallikrein inhibitory activity of plasma. The predominant role of $\mathrm{C} 1-\mathrm{INH}$ in the regulation of KKS activation in plasma is underscored by the fact that it simultaneously and efficiently inhibits FXIIa, PKA, and FXIa (Figure 1). The deficiency of $\mathrm{C} 1-\mathrm{INH}$ characteristic of patients with HAE-C1-INH causes plasma instability resulting in lower thresholds for KKS activation in response to dextran sulfate stimulation. ${ }^{25}$

\section{Kinin Signaling and Catabolism}

Biologically active kinins are peptide mediators predominantly generated by the enzymatic action of kallikreins on kininogen precursors. BK is a tissue hormone (autacoid) that is biologically active only close to its site of formation in a paracrine manner. With clinical effects similar to those of histamine, it is a vasodepressor that relaxes vascular smooth muscles and consequently lowers blood pressure and increases vascular permeability. ${ }^{26}$ It is a shortlived (30 seconds) nonapeptide (Arg-Pro-Pro-Gly-Phe-Ser-ProPhe-Arg) with potent (nanomolar) endothelium-dependent vasodi-

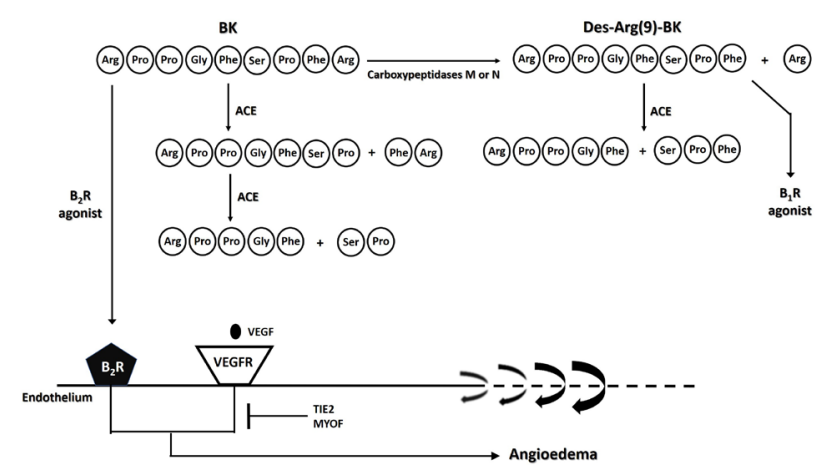

FIG. 2. BK signaling and catabolism. $B K$ is an agonist of $B_{2} R$. In physiological conditions, the metalloproteases carboxypeptidase $\mathrm{M}$ (CPM) and carboxypeptidase $\mathrm{N}$ remove the $\mathrm{C}$-terminal arginine residue from $\mathrm{BK}$ to release the octapeptide des-Arg9 $\mathrm{BK}$, which is a $\mathrm{B}_{1} \mathrm{R}$ agonist. des-Arg9 $B K$ is in turn cleaved by the membrane-bound peptidyl dipeptidase angiotensin-converting enzyme (ACE) to yield inactive peptides. ACE can also directly inactivate $\mathrm{BK}$ by cleavage after residues Phe5 and $\mathrm{PrO}_{7}$. $\mathrm{BK}$ binding to $\mathrm{B}_{2} \mathrm{R}$ ultimately promotes endothelial activation and angioedema by a phospholipase C- and inositol 1,4,5-trisphosphate-mediated mobilization of intracellular calcium reservoirs. This process requires coestimulation of VEGFR and is negatively regulated by the ANGPT1-Tie2 axis. lator activity. ${ }^{27,28} \mathrm{BK}$ binding on endothelial cells causes swelling, redness, and local heat, whereas its action on nonmyelinated afferent neurons directly produces pain and activates reflex responses by the autonomic nervous system. There is large evidence that BK also influences other cell types, such as enterocytes, fibroblasts, striated muscle cells, and cardiomyocytes, but the medical relevance of these observations is not yet clear. ${ }^{29}$

BK activity in plasma is transient owing to its rapid degradation by plasma peptidases and will accumulate only if its formation rate exceeds that of its breakdown. This in turn complicates the measurement of BK formation in plasma samples as a screening test for KKS activation in HAE, which is rarely performed..$^{30,31}$

The degradation of BK in plasma takes place in a sequential manner. The plasma metalloproteases carboxypeptidase $\mathrm{M}$ (CPM) and carboxypeptidase $\mathrm{N}$ (also known as kininase 1) remove the $\mathrm{C}$-terminal arginine residue from BK to leave an octapeptide (Arg-ProPro-Gly-Phe-Ser-Pro-Phe), des-Arg9 BK (des-Arg9-BK), which is then digested by the membrane-bound peptidyl dipeptidase angiotensin-converting enzyme (ACE) (also known as kininase 2) to yield the inactive pentapeptide Arg-Pro-Pro-Gly-Phe. ${ }^{32,33}$ Moreover, ACE can also directly cleave BK after residues $\mathrm{Phe}_{5}$ and $\mathrm{Pro}_{7}$ to produce only inactive peptides (Figure 2). The latter cleavage pattern can be performed by neutral endopeptidase (NEP), which is also a membrane-bound metalloprotease. Other enzymes acting mainly in the fluid phase that contributes to the cleavage and inactivation of $\mathrm{BK}$ are aminopeptidase $\mathrm{P}$ and dipeptidyl peptidase IV. However, in the context of HAE physiopathology, ACE and NEP seem to be the main proteins responsible for the systemic degradation of BK. The relevance of ACE in HAE is underscored by the fact that ACE inhibitors, broadly used for the treatment of arterial hypertension, cause BK-mediated angioedema in a small percentage of healthy individuals (from $0.1 \%$ to $1.6 \%$ according to different studies) and are a major environmental factor precipitating edema attacks in patients with HAE. ${ }^{1}$

BK and des-Arg9-BK bind to cellular surfaces through specific $G$ protein-coupled $B K$ receptors known as $B_{1} R$ and $B_{2} R$. BK binds predominantly to $B_{2} R$, whereas des-Arg9-BK is a poor $B_{2} R$ agonist but instead binds to $B_{1} R$, although with low affinity. There is recent evidence that Lys-BK (also known as kallidin), a form of BK with an additional lysine at the N-terminus released by tissue KLK1 mainly from LK, is also a direct agonist of $B_{2} R$, although the mechanism proposed for this interaction does not imply the activation of the contact phase..$^{30}$ The traditional model of HAE physiopathology is based on the stimulation of $\mathrm{B}_{2} \mathrm{R}$ by $\mathrm{BK}$ and is strongly supported by the following: (1) increased BK levels detected in the plasma of patients with HAE during attacks; (2) blockade of $\mathrm{B}_{2} \mathrm{R}$ abrogates the vasoactive effects of BK and prevents angioedema; (3) mice lacking $\mathrm{B}_{2} \mathrm{R}$ as well as $\mathrm{C} 1$-INH do not have evidence for increased vascular permeability, whereas $\mathrm{C} 1-\mathrm{INH}$-deficient mice with normal $B_{2} R$ suffer from increased vascular permeability; and (4) the low affinity of des-Arg9-BK for its $\mathrm{B}_{1} \mathrm{R}^{34-36}$ (Figure 2).

Both kinin-receptor interactions lead to phospholipase $\mathrm{C}$ activation and inositol 1,4,5-trisphosphate-mediated mobilization of intracellular calcium reservoirs, which ultimately cause the release of 
vasodilating agents and secondary mediators. These include nitric oxide, prostaglandin $\mathrm{I}_{2}$ (prostacyclin), endothelium-derived hyperpolarizing factor, and tissue plasminogen activator, which exert diverse physiological actions, including the regulation of vascular tone and local blood flow to organs, coagulation, fibrinolysis, and water-electrolyte balance. ${ }^{37}$

The nitric oxide released by BK-stimulated endothelial cells induces a rise of cyclic guanosine monophosphate levels in the vascular smooth muscle cells, whereas prostacyclin stimulates the production of cyclic adenosine monophosphate in the vascular endothelial and smooth muscle cells, both signals ultimately leading to the relaxation of the vascular musculature. Recent evidence obtained from patients with HAE-C1-INH has shown that BK is probably also associated with an increased release of VEGF-A and VEGF-C, angiopoietins 1 and 2, and secreted phospholipase A2.38,39

Interestingly, the $\mathrm{B}_{1} \mathrm{R}$ and $\mathrm{B}_{2} \mathrm{R}$ receptors present different patterns of expression and processing. Whereas $\mathrm{B}_{2} \mathrm{R}$ expression is constitutive in endothelial cells, smooth muscle cells, sensory neurons, and epithelial cells, $B_{1} R$ is inducible in vascular cells after tissue injury or cytokine stimulation. ${ }^{40}$ The injection of bacterial lipopolysaccharide in laboratory animals is a traditional model to sensitize the whole cardiovascular system to $B_{1} R$ agonists. Both receptors respond differently on kinin stimulation: the $\mathrm{B}_{2} \mathrm{R}$ is desensitized by a cycle of phosphorylation/endocytosis followed by recycling to the membrane, whereas the nonphosphorylable $B_{1} R$ is relatively resistant to desensitization and eventually translocates to caveolae. This in turn also determines that $\mathrm{B}_{1} \mathrm{R}$ and $\mathrm{B}_{2} \mathrm{R}$ have strikingly different half-lives. $\mathrm{B}_{2} \mathrm{R}$ is rapidly desensitized on interaction with $\mathrm{BK}$. In contrast, $\mathrm{B}_{1} \mathrm{R}$ takes several hours to become upregulated, but once present, $B_{1} R$ persists longer on the cell membrane. ${ }^{41,42}$ These differences in the temporal patterns of BK receptors expression have probably a deep influence on the physiopathology of HAE by influencing the response capacity of the endothelium and the duration of the edema attacks.

Local Manifestations of HAE and Systemic KKS Activation KKS is the final driver of angioedema development in patients with HAE. During angioedema episodes, HK is significantly cleaved, and $\mathrm{BK}$ levels are increased, whereas the plasma concentrations of contact system proteins, such as FXII, PK, and noncleaved HK, are decreased, and kallikrein-like enzymatic activity is significantly elevated in plasma during attacks. ${ }^{43-46}$ This central role of KKS is supported by the fact that all the genetic and molecular defects identified to date in patients with HAE (considering both the variant forms with and without C1-INH functional deficiency) seem to affect critical components controlling the activation of the CAS/ KKS system (C1-INH, FXII, HK, PLG) or the molecules involved in the transduction of BK signaling by endothelial cells (ANGPT1, MYOF).

HAE attacks seem to have both local and systemic features. On one hand, there is clear confirmation that a local process occurs, as shown by the presence of elevated kallikrein-like activity in the blisters from patients with $\mathrm{HAE}^{47}$ and the detectable BK levels in the venous compartments of affected tissues. ${ }^{48}$ On the other hand, changes measured in contact system parameters during HAE at- tacks are suggestive of a systemic activation process. Although the edema episodes are supposed to be initiated by local events of KKS activation on biological surfaces, the fluid phase is also expected to activate. Local bound PK will generate fluid phase FXIIa either by acting on fluid phase FXII molecules or by cleaving surface-bound FXIIa to FXIIf. FXIIf can activate PK to PKa only in the fluid phase, which in turn will activate more fluid phase FXII molecules. Furthermore, both PK and FXII, when bound to endothelial cells and activators, are largely protected from inactivation by $\mathrm{C} 1-\mathrm{INH}$. This strongly suggests that $\mathrm{C} 1-\mathrm{INH}$ prevents the propagation of KKS activation by inhibiting fluid-phase active proteases but has minor effects on surface-bound proteases.

This mechanistic explanation has led to an alternative pathogenic model for HAE-C1-INH (systemic hypothesis) involving both $\mathrm{B}_{1} \mathrm{R}$ and $\mathrm{B}_{2} \mathrm{R}$ receptors. According to this hypothesis, contact activation starts locally in response to different insults, such as minor traumas, and disseminates through the fluid phase. On local KKS activation, the decreased functional $\mathrm{C} 1-\mathrm{INH}$ levels in these patients are rapidly surpassed by small amounts of FXIIa, which results in systemic activation with massive HK cleavage and BK release. This in turn desensitizes endothelial $\mathrm{B}_{2} \mathrm{R}$ throughout the body, and edema develops exclusively at the sites of initial insult where cytokine release induces the local expression of $\mathrm{B}_{1} \mathrm{R}$ and CPM (which generates the $\mathrm{B}_{1} \mathrm{R}$ ligand des-Arg(9)-BK) (Figure 2). ${ }^{49}$ This model would help to explain some puzzling clinical features of HAE: on one hand, the fact that KKS activation in HAE is not associated with hypercoagulation disorders because fluid phase FXIIf does not have procoagulant activity, and on the other hand, the fact that $\mathrm{B}_{1} \mathrm{R}$-mediated stimulation better conforms to the slow onset and relapse dynamics of HAE attacks, because of the slow desensitization and cycling of $\mathrm{B}_{1} \mathrm{R}$, than that of $\mathrm{B}_{2} \mathrm{R}$. However, it still needs to be fully validated as a model for HAE-C1-INH, and the question remains as to how well it fits as an explanation for other HAE disease variants. All things considered, establishing a consensus pathological model for HAE is to date a point of open debate in the field.

\section{The Molecular Landscape of BK-Mediated Angioedema}

HAE-C1-INH was characterized at the molecular level almost 60 years $\mathrm{ago}^{2}$ and remained the only form of inherited BK-mediated angioedema ${ }^{34}$ until the description of the first nonhypocomplementemic (HAE-nC1-INH), estrogen-related HAE cohorts. ${ }^{50,51}$ The $21^{\text {st }}$ century has brought a considerable change in our understanding of HAE owing to the characterization of HAE-FXII, HAE-PLG, HAE-ANGPT1, and HAE-KNG1 disease variants. Although a complete physiopathological explanation is still needed for these new variants, growing evidence suggests that they are indeed BK-mediated. Moreover, the recent finding of myoferlin variants in patients with HAE-U expand the molecular picture of BK signaling and shed light on important interrelationships between the KKS and other physiological systems. The following sections provide a concise review of these functional links.

\section{HAE-C1-INH}

Decreased levels of antigenic (HAE-C1-INH type 1) or functional (HAE-C1-INH type 2) $\mathrm{C} 1$-INH results in a defective regulation of 
the KKS, the complement system, coagulation, and fibrinolysis. ${ }^{1}$ This is evidenced by the systemic activation of these systems as shown by the low levels of complements C4 and C2, FXII, PK, and $\mathrm{HK}$ during remission periods. At a serological level, there is also evidence for the activation of the coagulation pathway (increased levels of D-dimers, prothrombin fragment $1+2$, and thrombin-antithrombin complexes). ${ }^{52}$ However, both patients with HAE-C1INH and those with HAE-FXII do not appear to have an increased thrombotic risk, suggesting that pathological hyperactivity of the KKS does not appear to have thrombotic consequences on a clinical level. A broad review on the physiopathology of HAE-C1-INH can be found for example at reference number. ${ }^{53}$

\section{HAE-FXII}

Patients with HAE-FXII carry gain-of-function variants in the proline-rich domain of the $F 12$ gene, the most prevalent and best studied being the c.1032C > G (p.Thr309Lys) variant. This mutation has been particularly well studied at the molecular level. Pathophysiologically, p.Thr309Lys causes overactivation of the $\mathrm{KKS}$ in the fluid phase in different ways. It removes an O-glycosylation site, which probably has profound effects on FXII folding and results in a relaxed or open conformation that renders FXII susceptible to increased and/or noncanonical activation. It has been recently shown that this conformational change exposes proteolytic activation sites for thrombin and FXI and also increases the susceptibility of FXII to become activated by plasmin, ${ }^{54,55}$ all of which result in fluid phase activation of FXII. These findings also point to a complex pathological connection between KKS, coagulation, and fibrinolysis at the basis of HAE attacks in patients with HAE-FXII.

The expression of the $F 12$ gene is positively regulated by estrogens owing to the presence of estrogen-responsive elements in the promoter region, ${ }^{56}$ which explains the estrogen dependency of HAE symptoms in patients with HAE-FXII and is certainly involved in the pathogenesis of the remaining nonhypocomplementemic, HAE-nC1INH disease variants.

\section{HAE-PLG}

In 2018, Bork and colleagues identified the undescribed genetic variant c.988A > G (p.Lys330Glu) in the PLG gene from 14 patients with estrogen-dependent HAE-nC1-INH. ${ }^{57}$ The mutation is transmitted in an autosomal dominant manner and results in a novel type of dysplasminogenemia with pathological implications. ${ }^{58}$ To date, this variant has yet to be fully studied at a physiological level. However, its pathogenic nature is well supported by several observations.

First, the Lys330Glu variant strictly cosegregates with HAE symptoms in the studied series. Second, from a mechanistic perspective, the implication of plasmin generation in HAE-nC1-INH pathology is recognized by the effectiveness of antifibrinolytic agents (for example, tranexamic acid) as long-term prophylactic agents in patients with HAE-C1-INH, HAE-FXII, and HAE-PLG ${ }^{59-61}$ and the observation of low levels of PLG activator inhibitors 1 and 2 in HAE-FXII series, indicating a consumption by PLG activators. ${ }^{62}$
Interestingly, current measurements of BK synthesis and degradation in HAE-PLG blood samples by Marceau's group support an alternate kinin formation pathway involving KLK-1 activity and Lys-BK release in these patients. This alternative model would concord with some clinical features observed in patients with HAE-PLG: KLK-1 is highly expressed in salivary glands and human saliva, hypothetically correlating with the clinical presentation of HAE-PLG that includes the swelling of the tongue, lips, and contiguous throat tissues. These results are very recent and intriguing and might open an innovative line of research in HAE, but further research is needed to assess kinin formation heterogeneity. ${ }^{30}$

\section{HAE-ANGPT1}

Also, in 2018, Baffuno et al. ${ }^{63}$ have reported the finding of the c. $807 \mathrm{G}>\mathrm{T}$ (p.Ala119Ser) autosomal dominant variant in the gene encoding angiopoietin $1(A N G P T 1)$ in a single Italian family previously diagnosed with $\mathrm{U}-\mathrm{HAE}$.

ANGPT1 is the ligand for the TIE2 receptor, which is expressed in vascular endothelial cells and a subset of hematopoietic cells. The ANGPT1-TIE2 signaling axis inhibits the effects of several permeability factors, including VEGF and $\mathrm{BK},{ }^{64}$ and contributes to the regulation of the endothelial barrier function. The Ala119Ser pathogenic variant reduces the amounts of ANGPT1 in the plasma and impairs the assembly of ANGPT1 multimers, leading to reduced binding to the TIE2 receptor owing to a mechanism of haploinsufficiency. ${ }^{65}$

\section{HAE-KNG1}

HAE-KNG1 is an estrogen-related form of HAE-nC1-INH caused by the c.1136T > A (p.Met379Lys) variant in the KNG1 gene. The affected residue is present in both $\mathrm{HK}$ and LK proteins and affects the N-terminal cleavage site of BK. A total of 2 alternative hypotheses have already been proposed as physiopathological mechanisms for HAE-KNG1. On one hand, as Met379Lys modifies the cleavage sites of $\mathrm{HK}$ and $\mathrm{LK}$, it could result in aberrant forms of BK (released by PKa from HK) and Lys-BK (released by tissue kallikrein from LK) and interfere in their inactivation process by peptidases. Alternatively, it might increase the accessibility of $\mathrm{PKa}$ for its cleavage sites in $\mathrm{HK}$, resulting in enhanced BK release. ${ }^{66}$

\section{HAE-MYOF}

This novel subtype of HAE-nC1-INH was described in 2020 in a single Italian family diagnosed with U-HAE. Affected members from the family bear the c.651G $>\mathrm{T}$ (p.Arg217Ser) variant in the MYOF gene encoding myoferlin 1, a $230-\mathrm{kDa}$ type-II integral membrane protein, located at the plasma membrane of endothelial cells that regulates VEGF signaling by preventing the ubiquitination and degradation of its receptor VEGFR-2. Functional studies performed in cell lines have shown that the Arg217Ser variant increases the levels of VEGFR-2 in response to VEGF stimuli by modifying the subcellular distribution of the protein and improving its localization on the plasma membrane. ${ }^{67}$

Both HAE-MYOF and HAE-ANGPT1 are related in terms of involving pathological mechanisms that do not directly involve increased BK generation but instead involves altered intracellular 
transduction by closely related pathways. Besides, both disease subtypes have yet to be replicated in other HAE cohorts and better characterized at the functional level.

\section{CONCLUSION}

In recent years, increasing insight into the pathogenesis of HAE and the access to next-generation sequencing technologies have led to the discovery of new disease variants, which in turn has improved the diagnostic and therapeutic management of patients with HAE. The loss of endothelial barrier function during HAE episodes originates from a loss of homeostatic imbalance, which may involve different extra and intracellular pathways. Whereas the central pathogenic position of the KKS in HAE is well established, some important questions remain. The roles played by novel drivers of HAE (such as ANGPT1 and MYOF) and their particular connections with BK signaling, the precise pathogenic mechanisms of new HAE disease variants, or the impacts of heterogeneous kinin formation are still the focus of active research.

Conflict of Interest: The author has no conflicts of interest to declare.

Funding: Alberto López Lera is supported by the Centre for Biomedical Network Research on Rare Diseases (CIBERER). This work was funded by grant (ER19P7AC7541) - ACCI18-04 from CIBERER and Complemento II-CM network (B2017/BMD3673).

\section{REFERENCES}

1. Caballero T, Baeza ML, Cabañas R, et al. Consensus statement on the diagnosis, management, and treatment of angioedema mediated by bradykinin. Part I. Classification, epidemiology, pathophysiology, genetics, clinical symptoms, and diagnosis. J Investig Allergol Clin Immunol. 2011;21(5):333-347; quiz follow 347.

2. Donaldson VH, Evans RR. A biochemical abnormality in herediatry angioneurotic edema: absence of serum inhibitor of C' 1-ESTERASE. Am J Med. 1963;35:37-44 [Crossref]

3. Marcelino-Rodriguez I, Callero A, Mendoza-Alvarez A, et al. Bradykinin-mediated angioedema: an update of the genetic causes and the impact of genomics. Front Genet. 2019;10:900. [Crossref]

4. Schmaier AH. The contact activation and kallikrein/kinin systems: pathophysiologic and physiologic activities. J Thromb Haemost. 2016 J;14(1):28-39. [Crossref]

5. Renné T. The procoagulant and proinflammatory plasma contact system. Semin Immunopathol. 2012;34(1):31-41. [Crossref]

6. Weidmann H, Heikaus L, Long AT, Naudin C, Schlüter H, Renné T. The plasma contact system, a protease cascade at the nexus of inflammation, coagulation and immunity. Biophys Acta Mol Cell Res. 2017;1864(11 Pt B):2118-2127. [Crossref]

7. Pathak M, Wilmann P, Awford J, et al. Coagulation factor XII protease domain crystal structure. J Thromb Haemost. 2015;13(4):580-591. [Crossref]

8. Kaplan AP, Joseph K. Pathogenic mechanisms of bradykinin mediated diseases: dysregulation of an innate inflammatory pathway. Adv Immunol. 2014;121:41-89. [Crossref]

9. Ghebrehiwet B, Silverberg M, Kaplan AP. Activation of the classical pathway of complement by Hageman factor fragment. $J$ Exp Med. 1981;153(3):665-676. [Crossref]

10. Mandle RJ, Colman RW, Kaplan AP. Identification of prekallikrein and high-molecular-weight kininogen as a complex in human plasma. Proc Natl Acad Sci U S A. 1976;73(11):4179-4183. [Crossref]

11. Scott CF, Colman RW. Function and immunochemistry of prekallikrein-high molecular weight kininogen complex in plasma. J Clin Invest. 1980;65(2):413-421.

12. Tankersley DL, Finlayson JS. Kinetics of activation and autoactivation of human factor XII. Biochemistry. 1984;23(2):273-279. [Crossref]

13. Irmscher $\mathrm{S}$, Döring $\mathrm{N}$, Halder LD, et al. Kallikrein Cleaves $\mathrm{C} 3$ and Activates Complement. J Innate Immun. 2018;10(2):94-105. [Crossref]

14. Colman RW, Schmaier AH. Contact system: A vascular biology modulator with anticoagulant, profibrinolytic, antiadhesive, and proinflammatory attributes. Blood 1997;90(10):3819-3843. [Crossref]
15. Lalmanach G, Naudin C, Lecaille F, Fritz H. Kininogens: More than cysteine protease inhibitors and kinin precursors. Biochimie. 2010 ;92(11):1568-1579. [Crossref]

16. Herwald H, Dedio J, Kellner R, Loos M, Muller-Esterl W. Isolation and characterization of the kininogen-binding protein $\mathrm{p} 33$ from endothelial cells. Identity with the gC1q receptor. J Biol Chem. 1996;271(22):13040-13047. [Crossref]

17. Mahdi F, Madar ZS, Figueroa CD, Schmaier AH. Factor XII interacts with the multiprotein assembly of urokinase plasminogen activator receptor, $\mathrm{gClqR}$, and cytokeratin 1 on endothelial cell membranes. Blood. 2002;99(10):3585-3596. [Crossref]

18. Colman RW, Pixley RA, Najamunnisa S, et al. Binding of high molecular weight kininogen to human endothelial cells is mediated via a site within domains 2 and 3 of the urokinase receptor. J Clin Invest. 1997;100(6):1481-1487. [Crossref]

19. Kaplan AP, Austen KF. A pre-albumin activator of prekallikrein. J Immunol. 1970;105(4):802-811.

20. Shariat-Madar Z, Mahdi F. Schmaier A. Identification and characterization of prolylcarboxypeptidase as an endothelial cell prekallikrein activator. $J$ Biol Chem. 2002;277(20):17962-17969. [Crossref]

21. Adams GN, LaRusch GA, Stavrou E, et al. Murine prolylcarboxypeptidase depletion induces vascular dysfunction with hypertension and faster arterial thrombosis. Blood. 2011;117:3929-3937. [Crossref]

22. Joseph K, Tholanikunnel BG, Kaplan AP. Heat shock protein 90 catalyzes activation of the prekallikrein-kininogen complex in the absence of factor XII. Proc Natl Acad Sci U S A. 2002;99(2):896-900. [Crossref]

23. Joseph K, Tholanikunnel BG, Kaplan AP. Activation of the bradykinin-forming cascade on endothelial cells: a role for heat shock protein 90. Int Immunopharmacol. 2002;2(13-14):1851-1859. [Crossref]

24. Maas C, Schiks B, Strangi RD, et al. Identification of fibronectin type I domains as amyloid-binding modules on tissue-type plasminogen activator and three homologs. Amyloid. 2008;15(3):166-180. [Crossref]

25. Cameron CL, Fisslthaler B, Sherman A, Reddigari S, Silverberg M. Studies on contact activation: effects of surface and inhibitors. Med Prog Technol. 1989;15(1-2):5362 .

26. Bas M, Adams V, Suvorava T, Niehues T, Hoffmann TK, Kojda G. Nonallergic angioedema: role of bradykinin. Allergy. 2007;62(8):842-856. [Crossref]

27. Howl J, Payne S. Bradykinin receptors as a therapeutic target. Expert Opin Ther Targets. 2003;7(2):277-285. [Crossref]

28. Marceau F, Hess JF, Bachvarov DR. The B1 receptors for kinins. Pharmacol Rev 1998;50(3):357-386.

29. Marceau F, Regoli D. Bradykinin receptor ligands: therapeutic perspectives. Nat Rev Drug Discov. 2004;3(10):845-852. [Crossref]

30. Marceau F, Rivard GE, Gauthier JM, et al. Measurement of bradykinin formation and degradation in blood plasma: relevance for acquired angioedema associated with angiotensin converting enzyme inhibition and for hereditary angioedema due to factor XII or plasminogen gene variants. Front Med (Lausanne). 2020;7:358. [Crossref]

31. de Maat S, Björkqvist J, Suffritti C, Wiesenekker CP, Nagtegaal W, Koekman A, van Dooremalen S, Pasterkamp G, de Groot PG, Cicardi M, Renné T, Maas C. Plasmin is a natural trigger for bradykinin production in patients with hereditary angioedema with factor XII mutations. J Allergy Clin Immunol. 2016;138(5):1414-1423.e9. [Crossref]

32. Sheikh I, Kaplan A. Studies of the digestion of bradykinin, Lys-bradykinin, and des-Arg9-bradykinin by angiotensin converting enzyme. Biochem Pharmacol. 1986;35(12):1951-1956. [Crossref]

33. Sheikh I, Kaplan A. Studies of the digestion of bradykinin, lysyl bradykinin, and kinin-degradation products by carboxypeptidases A, B, and N. Biochem Pharmacol. 1986;35(12):1957-1963. [Crossref]

34. Nussberger J, Cugno M, Amstutz C, Cicardi M, Pellacani A, Agostoni A. Plasma bradykinin in angioedema. Lancet. 1998;351(9117):1693-1697. [Crossref]

35. Griesbacher T, Sametz W, Legat FJ, Diethart S, Hammer S, Juan H. Effects of the non-peptide B2 antagonist FR173657 on kinin-induced smooth muscle contraction and relaxation, vasoconstriction and prostaglandin release. $\mathrm{Br} J$ Pharmacol. 1997;121(3):469-476. [Crossref]

36. Griesbacher T, Legat FJ. Effects of FR173657, a non-peptide B2 antagonist, on kinin-induced hypotension, visceral and peripheral oedema formation and bronchoconstriction. Br J Pharmacol. 1997;120(5):933-939. [Crossref]

37. Su JB. Kinins and cardiovascular diseases. Curr Pharm Des. 2006;12(26):3423-3435 [Crossref] 
38. Loffredo S, Bova M, Suffritti C, et al. Elevated plasma levels of vascular permeability factors in C1 inhibitor-deficient hereditary angioedema. Allergy. 2016;71(7):989-996. [Crossref]

39. Loffredo S, Ferrara AL, Bova M, et al. Secreted Phospholipases A(2) in Hereditary Angioedema With C1-Inhibitor Deficiency. Front Immunol. 2018;9:1721. [Crossref]

40. Koumbadinga GA, Désormeaux A, Adam A, Marceau F. Effect of interferon- $\gamma$ on inflammatory cytokine-induced bradykinin B1 receptor expression in human vascular cells. Eur J Pharmacol. 2010;647(1-3):117-125. [Crossref]

41. Enquist J, Sandén C, Skröder C, Mathis SA, Leeb-Lundberg LM. Kinin-stimulated $\mathrm{B} 1$ receptor signaling depends on receptor endocytosis whereas $\mathrm{B} 2$ receptor signaling does not. Neurochem Res. 2014;39(6):1037-1047. [Crossref]

42. Wolsing DH, Rosenbaum JS. Bradykinin-stimulated inositol phosphate production in NG108-15 cells is mediated by a small population of binding sites which rapidly desensitize. J Pharmacol Exp Ther. 1991;257(2):621-633.

43. Nussberger J, Cugno M, Cicardi M. Bradykinin-mediated angioedema. N Engl J Med. 2002;347(8):621-622. [Crossref]

44. Cugno M, Cicardi M, Coppola R, Agostoni A. Activation of factor XII and cleavage of high molecular weight kininogen during acute attacks in hereditary and acquired C1-inhibitor deficiencies. Immunopharmacology 1996;33(1-3):361-364. [Crossref]

45. Suffritti C, Zanichelli A, Maggioni L, Bonanni E, Cugno M, Cicardi M. High-molecular-weight kininogen cleavage correlates with disease states in the bradykinin-mediated angioedema due to hereditary C1-inhibitor deficiency. Clin Exp Allergy. 2014;44(12):1503-1514. [Crossref]

46. Defendi F, Charignon D, Ghannam A, et al. Enzymatic assays for the diagnosis of bradykinin-dependent angioedema. PLoS One. 2013;8(8):e70140. [Crossref]

47. Curd J, Prograis LJ, Cochrane C. Detection of active kallikrein in induced blister fluids of hereditary angioedema patients. J Exp Med. 1980;152(3):742-747. [Crossref]

48. Nussberger J, Cugno M, Cicardi M, Agostoni A. Local bradykinin generation in hereditary angioedema. J Allergy Clin Immunol 1999;104(6):1321-1322. [Crossref]

49. Hofman ZL, Relan A, Zeerleder S, Drouet C, Zuraw B, Hack CE. Angioedema attacks in patients with hereditary angioedema: Local manifestations of a systemic activation process. J Allergy Clin Immunol. 2016;138(2):359-366. [Crossref]

50. Bork K, Barnstedt SE, Koch P, Traupe H. Hereditary angioedema with normal C1-inhibitor activity in women. Lancet. 2000;356(9225):213-217. [Crossref]

51. Binkley KE, Davis A 3rd. Clinical, biochemical, and genetic characterization of a novel estrogen-dependent inherited form of angioedema. J Allergy Clin Immunol. 2000;106(3):546-550. [Crossref]

52. Cugno M, Zanichelli A, Bellatorre AG, Griffini S, Cicardi M. Plasma biomarkers of acute attacks in patients with angioedema due to C1-inhibitor deficiency. Allergy. 2009;64(2):254-257. [Crossref]
53. Kaplan A. Enzymatic pathways in the pathogenesis of hereditary angioedema: the role of C1 inhibitor therapy. J Allergy Clin Immunol. 2010;126(5):918-925. [Crossref]

54. de Maat S, Björkqvist J, Suffritti C, et al. Plasmin is a natural trigger for bradykinin production in patients with hereditary angioedema with factor XII mutations. J Allergy Clin Immunol. 2016;138(5):1414-1423.e9. [Crossref]

55. Ivanov I, Matafonov A, Sun MF, et al. A mechanism for hereditary angioedema with normal $\mathrm{C} 1$ inhibitor: an inhibitory regulatory role for the factor XII heavy chain. Blood. 2019;133(10):1152-1163. [Crossref]

56. Farsetti A, Misiti S, Citarella F, et al. Molecular basis of estrogen regulation of Hageman factor XII gene expression. Endocrinology. 1995;136(11):5076-5083. [Crossref]

57. Bork K, Wulff K, Steinmüller-Magin L, et al. Hereditary angioedema with a mutation in the plasminogen gene. Allergy. 2018;73(2):442-450. [Crossref]

58. Dewald G. A missense mutation in the plasminogen gene, within the plasminogen kringle 3 domain, in hereditary angioedema with normal $\mathrm{C} 1$ inhibitor. Biochem Biophys Res Commun. 2018;498(1):193-198. [Crossref]

59. Sheffer A, Austen K, Rosen F. Tranexamic acid therapy in hereditary angioneurotic edema. N Engl J Med. 1972; 1972287(9):452-454.

60. Bork K, Wulff K, Witzke G, Hardt J. Treatment for hereditary angioedema with normal C1-INH and specific mutations in the F12 gene (HAE-FXII). Allergy. 2017;72(2):320-324. [Crossref]

61. Bork K. Acquired and hereditary forms of recurrent angioedema: Update of treatment Allergol Select. 2018;2(1):121-131. [Crossref]

62. Joseph K, Tholanikunnel BG, Wolf B, Bork K, Kaplan AP. Deficiency of plasminogen activator inhibitor 2 in plasma of patients with hereditary angioedema with normal $\mathrm{C} 1$ inhibitor levels. J Allergy Clin Immunol. 2016;137(6):1822-1829.e1. [Crossref]

63. Bafunno V, Firinu D, D'Apolito M, et al. Mutation of the angiopoietin-1 gene (ANGPT1) associates with a new type of hereditary angioedema. J Allergy Clin Immunol. 2018;141(3):1009-1017. [Crossref]

64. Baffert F, Le T, Thurston G, McDonald DM. Angiopoietin-1 decreases plasma leakage by reducing number and size of endothelial gaps in venules. Am J Physiol Heart Circ Physiol. 2006;290(1):H107-118. [Crossref]

65. D'Apolito M, Santacroce R, Colia AL, Cordisco G, Maffione AB, Margaglione M. Angiopoietin-1 haploinsufficiency affects the endothelial barrier and causes hereditary angioedema. Clin Exp Allergy. 2019;49(5):626-635. [Crossref]

66. Bork K, Wulff K, Rossmann $\mathrm{H}$, et al. Hereditary angioedema cosegregating with a novel kininogen 1 gene mutation changing the $\mathrm{N}$-terminal cleavage site of bradykinin. Allergy. 2019;74(12):2479-2481. [Crossref]

67. Ariano A, D'Apolito M, Bova M, et al. A myoferlin gain-of-function variant associates with a new type of hereditary angioedema. Allergy. 2020;75(11):2989-2992. [Crossref] 